\title{
NEW DIVISION ALGEBRAS
}

BY L. E. DICKSON

1. Introduction. No technical acquaintance with linear algebras is presupposed in this note. We consider only linear algebras for which multiplication is associative. As with quaternions, an algebra $A$ is called a division algebra if every element $\neq 0$ of $A$ has an inverse in $A$. A division algebra $A$ over a field $F$ is called normal if the numbers of $F$ are the only elements of $A$ which are commutative with every element of $A$.

In a paper recently offered to the Transactions of this Society, A. A. Albert determined all normal division algebras of order 16 and found a new type. The object of this note is to derive from mild assumptions the corresponding type of normal division algebras $A$ of order $4 p^{2}$, where $p$ is a prime. We shall first draw simple conclusions from an initial assumption.*

Assumption 1. Let $A$ contain an element $i_{1}$ satisfying an equation $f\left(\omega^{2}\right)=0$ of degree $2 p$ with only even powers of $\omega$, whose coefficients are in $F$, that of $\omega^{2 p}$ being unity, and which is irreducible in $F$, such that the polynomials in $i_{1}$ are the only elements of $A$ which are commutative with every element of $A$.

2. Lemma 1. Let an element $i_{2}$ of $A$ be commutative with $I=i_{1}^{2}$, but not with $i_{1}$ itself. The algebra $S$ generated by $i_{1}$ and $i_{2}$ is of order $4 p$. It may be regarded as an algebra of order 4 with the basis $1, i_{1}, i_{2}, i_{1} i_{2}$ over $F(I)$; this algebra is normal. In other words, the polynomials in I are the only elements of $S$ which are commutative with every element of $S$.

Let $K$ denote the field composed of all those elements of

* Except for the requirement concerning even powers of $\omega$, Assumption 1 is proved in the writer's Algebren und ihre Zahlentheorie, Zürich, 1927, pp. 262-3. 
$S$ which are commutative with every element of $S$. If $K$ is of order $k$ and $S$ is of order $s$ over $F$, then $S$ is a normal division algebra of order $n^{2}$ over $K$, where $s=n^{2} k$. Since $K$ contains the root $I$ of an equation of degree $p$ irreducible in $F$, the subfield $F(I)$ is of order $p$, whence $k$ is a multiple of $p$.

Since $i_{2}$ is not commutative with $i_{1}, i_{2}$ is not a polynomial in $i_{1}$ and hence is not a rational function of $i_{1}$. Thus

$$
i_{1}^{j}, i_{1}^{j} i_{2}, \quad(j=0,1, \cdots, 2 p-1),
$$

are linearly independent with respect to $F$. Hence $s \geqq 4 p$. Since $S$ and $A$ are normal over different fields $K$ and $F$, $S \neq A$. Thus $s$ is a divisor $<4 p^{2}$ of $4 p^{2}$. First, let $p>2$. If $s$ is not divisible by $p^{2}$, then $s=4 p$. But if $s$ is divisible by $p^{2}$, either $s=2 p^{2}$, or $s=p^{2}$ and $p>4$. If $p=2$, evidently $s=8=4 p$.

If either $s=p^{2}, p>4$, or $s=2 p^{2}, p>2$, then $s=n^{2} k$ and the divisibility of $k$ by $p$ show that $n=1, S=K$, contrary to the fact that $i_{2}$ is not commutative with $i_{1}$.

Hence $s=4 p=n^{2} k$, whence $n=2, k=p$. Thus $K=F(I)$ and $S$ is a normal algebra of order 4 over $F(I)$. The $4 p$ elements (1) form a basis of $S$ over $F$.

3. Lemma 2. Any element of $A$ which is commutative with $I=i_{1}^{2}$ belongs to $S$.

Any element not in $S$ extends $S$ to a division subalgebra whose order exceeds $4 p$, is a multiple of $4 p$, and is a divisor of $4 p^{2}$. Hence it extends $S$ to $A$ itself (of order $4 p^{2}$ ).

Suppose that $e$ is commutative with $I$ and is not in $S$. Since $I$ is commutative with every element of $S$ and with $e$, which extends $S$ to $A, I$ is commutative with every element of $A$. Since $I$ is not in $F$, this contradicts the hypothesis that $A$ is normal over $F$.

4. Assumption 2. Let $A$ contain elements $i_{1}$ and $z$ such that $i_{1}$ satisfies Assumption 1 and such that

$$
i_{2}=z i_{1} z^{-1}, i_{3}=z i_{2} z^{-1}, \cdots, i_{p}=z i_{p-1} z^{-1}
$$


are all commutative with $I=i_{1}{ }^{2}$, while $i_{2}$ is not commutative with $i_{1}$, and $i_{2}^{2} \neq I$.

Since $z I z^{-1}=i_{2}{ }^{2} \neq I, z$ is not commutative with $I$ and hence is not in $S$. By $\S 3, z$ extends $S$ to $A$. Since (1) gives a basis of $S$, every element of $S$ is of the form

$$
G=p\left(i_{1}\right)+q\left(i_{1}\right) i_{2} .
$$

Then

$$
G^{\prime}=z G z^{-1}=p\left(i_{2}\right)+q\left(i_{2}\right) i_{3} .
$$

For $p \geqq 3, i_{3}$ is commutative with $i_{1}{ }^{2}$ and hence is in $S$. Thus

$$
z G=G^{\prime} z, G^{\prime} \text { in } S .
$$

5. Lemma 3. $i_{1}^{2}, \cdots, i_{p}^{2}$ are all distinct.

Suppose that $i_{r+1}^{2}=i_{1}^{2}$, where $r$ is one of $2,3, \cdots, p-1$. Then

$$
z^{r} i_{1}^{2} z^{-r}=i_{r+1}^{2}=i_{1}{ }^{2},
$$

whence $z^{r}$ is commutative with $i_{1}^{2}$ and is in $S$. Using also (5), we see that every element of the algebra $A$ obtained by extending $S$ by $z$ is of the form

$$
H_{0}+H_{1} z+\cdots+H_{r-1} z^{r-1},
$$

where each $H$ is in $S$. Since $S$ is of order $4 p$, the order of $A$ is $\leqq 4 p \cdot r<4 p^{2}$. But $A$ is of order $4 p^{2}$.

Suppose that $i_{r+s}^{2}=i_{s}^{2}(r>0, s>1)$. These are the transforms of $i_{r+s-1}^{2}$ and $i_{s-1}^{2}$ by $z$. Hence the latter are equal. After $s-1$ such steps, we get $i_{r+1}^{2}=i_{1}^{2}$, just proved impossible.

6. LEMma 4. We have the following identity:

$$
f(\epsilon) \equiv\left(\epsilon-i_{p}^{2}\right) \cdots\left(\epsilon-i_{2}^{2}\right)\left(\epsilon-i_{1}^{2}\right) .
$$

Note that

$$
i_{r} \text { is commutative with } i_{r+1}, \cdots, i_{p}, \quad(r=1, \cdots, p-1) .
$$

This is true by Assumption 2 if $r=1$. To proceed by induction, let (7) hold when $r=j$, whence $i_{j}^{2}$ is commutative 
with $i_{k}$ for $k \geqq j+1$. Transformation by $z$ shows that $i_{j+1}{ }^{2}$ is commutative with $i_{k+1}$, whence (7) holds when $r=j+1$.

Write $v_{j}$ for $i_{7}^{2}$. As a special case of $(7), v_{1}, \cdots, v_{p}$ are commutative. The indeterminate $\epsilon$ is commutative with every quantity of $A$. Thus $z$ transforms $f(\epsilon)$ into itself. But $f\left(v_{1}\right)=0$. Hence by $(2), f\left(v_{2}\right)=0, \cdots, f\left(v_{p}\right)=0$. Let

$$
\begin{array}{rr}
f(\epsilon)= & \sum_{j=0}^{p} a_{j} \epsilon^{p-j}, q(\epsilon)=\sum_{j=0}^{p-1} c_{j} \epsilon^{p-1-i}, a_{0}=c_{0}=1, \\
c_{j}=a_{j}+c_{\jmath-1} v_{1}, & (j=1, \cdots, p) .
\end{array}
$$

Then, since $v_{1}$ is commutative with $\epsilon$,

$$
f(\epsilon) \equiv q(\epsilon)\left(\epsilon-v_{1}\right)+c_{p} .
$$

By induction on $r$,

$$
c_{r}=\sum_{j=0}^{r} a_{j} v_{1}^{r-j}, \quad c_{p}=f\left(v_{1}\right)=0 .
$$

Since $v_{i}$ is commutative with $v_{1}$, we obtain a true equality from (8) by replacing $\epsilon$ by $v_{i}$. Thus $0=q\left(v_{i}\right)\left(v_{i}-v_{1}\right)$. The second factor is not zero if $i \geqq 2$. In our division algebra we therefore have $q\left(v_{i}\right)=0$ when $i \geqq 2$.

We may repeat this argument with $f$ and $v_{1}$ replaced by $q$ and $v_{2}$. Hence $q(\epsilon) \equiv r(\epsilon)\left(\epsilon-v_{2}\right)$, in which the coefficients of $r(\epsilon)$ are polynomials in $v_{1}$ and $v_{2}$. Since they are commutative with $v_{j}, 0=r\left(v_{j}\right)\left(v_{j}-v_{2}\right)$. Hence $r\left(v_{j}\right)=0$ when $j \geqq 3$.

Proceeding similarly, we ultimately obtain

$$
f(\epsilon) \equiv\left(\epsilon-v_{p}\right) \cdots\left(\epsilon-v_{2}\right)\left(\epsilon-v_{1}\right) .
$$

7. THEOREM 1. $f(\epsilon)=0$ is a cyclic equation.

By (6), $i_{1}^{2}+\cdots+i_{p}^{2}$ is a number of $F$ and hence is transformed into itself by $z$. But $z$ transforms $i_{1}^{2}$ into $i_{2}^{2}$, $\cdots, i_{p-1}^{2}$ into $i_{p}^{2}$. Hence $z$ must transform $i_{p}^{2}$ into $i_{1}^{2}$. Since $z^{p-2}$ transforms $i_{2}^{2}$ into $i_{p}^{2}, z^{p-1}$ transforms $i_{2}^{2}$ into $i_{1}^{2}$ and evidently transforms $i_{1}$ into $i_{p}$. Hence $z^{p-1}$ transforms 
$i_{2}^{2} i_{1}$ and $i_{1} i_{2}^{2}$ into $i_{1}^{2} i_{p}$ and $i_{p} i_{1}^{2}$. The latter are equal by by Assumption 2. Hence the former are equal. Since $i_{2}{ }^{2}$ is therefore commutative with both generators $i_{1}$ and $i_{2}$ of $S$, it is commutative with every element of $S$. By Lemma 1 , $i_{2}{ }^{2}=\theta\left(i_{1}^{2}\right)$, where $\theta$ is a polynomial with coefficients in $F$. Transformation by $z$ gives

$$
i_{3}{ }^{2}=\theta\left(i_{2}{ }^{2}\right)=\theta\left[\theta\left(i_{1}{ }^{2}\right)\right]=\theta^{2}\left(i_{1}{ }^{2}\right),
$$

if $\theta^{r}(k)$ denotes the $r$ th iterative of $\theta(k)$ and not its $r$ th power. By induction,

$$
i_{r+1}^{2}=\theta^{r}\left(i_{1}^{2}\right) .
$$

Take $r=p-1$ and transform by $z$. Hence

$$
i_{1}^{2}=\theta^{p-1}\left(i_{2}^{2}\right)=\theta^{p}\left(i_{1}^{2}\right) .
$$

Since $f(\epsilon)=0$ has these properties, it is cyclic.

8. Theorem 2. Every element of $A$ can be expressed in one and only one way in the form

$$
A_{0}+A_{1} z+\cdots+A_{p-1} z^{p-1},
$$

where each $A_{j}$ is in $S$. The product any two sums (11) can be expressed as a third such sum by means of

$$
z G=G^{\prime} z, \quad z^{p}=s,
$$

where $G, G^{\prime}$, s are all in $S$ and are defined in (4), (5).

Since $z^{p-1}$ transforms $i_{1}{ }^{2}$ into $i_{p}^{2}$, and $z$ transforms the latter into the former, $z^{p}$ is commutative with $i_{1}{ }^{2}$ and hence is in $S$. By means of (12), every element of $A$ (to which $z$ extends $S$ ) can be expressed in the form (11). Since $S$ and $A$ are of orders $4 p$ and $4 p^{2}$, two polynomials (11) are distinct unless identical.

9. THEOREM 3. $S$ is an algebra of generalized quaternions over $F(I)$ with the basis $1, i_{1}, y, i_{1} y$, where $y=i_{1} i_{2}-i_{2} i_{1}$.

Since $i_{2}$ is not commutative with $i_{1}, y \neq 0$. Since $i_{2}$ is commutative with $i_{1}^{2}$, 


$$
y i_{1}=-i_{1} y .
$$

Thus $y$ is not commutative with $i_{1}$ and hence is not a polynomial in $i_{1}$. We may therefore replace the basis (1) of $S$ over $F$ by $i_{1}{ }^{j}, i_{1}{ }^{j} y$. Thus $S$ has the basis in Theorem 3 .

By $\S 7, i_{2}{ }^{2}$ is commutative with $i_{1}$. Hence

$$
r=i_{1} i_{2}+i_{2} i_{1}
$$

is commutative with $i_{2}$. Since $i_{2}$ is commutative with $I=i_{1}^{2}, r i_{1}=i_{1} r$. Hence $r$ is commutative with every element of $S$. Thus $r$ is a polynomial $P(I)$ in $I$. We have

$$
2 i_{1} i_{2}=P(I)+y, 2 i_{2} i_{1}=P(I)-y .
$$

But $y$ is commutative with $I$. Hence

$$
4 i_{1} i_{2}^{2} i_{1}=P^{2}-y^{2} .
$$

Since $i_{2}{ }^{2}$ is commutative with $i_{1}$,

$$
y^{2}=[P(I)]^{2}-4 I \theta(I) .
$$

This fact that $y^{2}$ is a polynomial in $I$ and relation (13) together show that $S$ is an algebra of generalized quaternions over $F(I)$.

The University of Chicago 